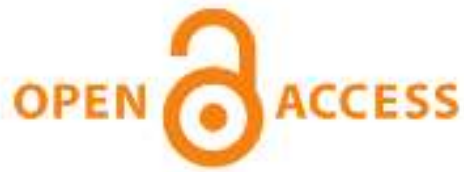

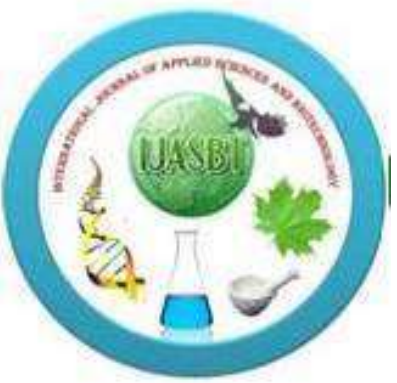 \\ International Journal of Applied Sciences and Biotechnology
}

\author{
A Rapid Publishing Journal
}

ISSN 2091-2609

\section{Indexing and Abstracting}

CrossRef, Google Scholar, Global Impact Factor, Genamics, Index Copernicus, Directory of Open Access Journals, WorldCat, Electronic Journals Library (EZB), Universitätsbibliothek Leipzig, Hamburg University, UTS (University of Technology, Sydney): Library, International Society of Universal Research in Sciences (EyeSource), Journal Seeker, WZB, Socolar, BioRes, Indian Science, Jadoun Science, JourInformatics, Journal Directory, JournalTOCs, Academic Journals Database, Journal Quality Evaluation Report, PDOAJ, Science Central, Journal Impact Factor, NewJour, Open Science Directory, Directory of Research Journals Indexing, Open Access Library, International Impact Factor Services, SciSeek, Cabell's Directories, Scientific Indexing Services, CiteFactor, UniSA Library, InfoBase Index, Infomine, Getinfo, Open Academic Journals Index, HINARI, etc.

\section{CODEN (Chemical Abstract Services, USA): IJASKD}

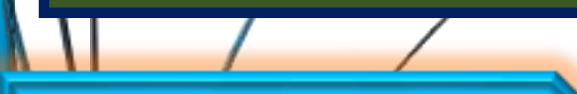

Vol-3(2) June, 2015

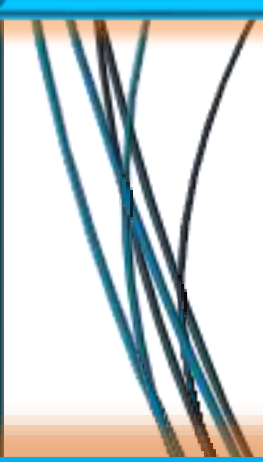

\section{Available online at:}

http://www.ijasbt.org

$\&$

http://www.nepjol.info/index.php/IJASBT/index

Impact factor*: $\mathbf{1 . 4 2 2}$

Scientific Journal Impact factor\#: 3.419

SEM-Biotech

Publishing 


\title{
WIDE SPREAD PREVALENCE OF $\beta$-LACTAM RESISTANCE AMONG BACTERIAL SPECIES OBTAINED FROM NON-CLINICAL SAMPLES
}

\author{
Shivangi Bhatt ${ }^{{ }^{*}}$ and Neepa Pandhi ${ }^{2}$ \\ ${ }^{1}$ Biochemistry Department, Shree M\& N Virani Science College, Rajkot, India \\ ${ }^{2}$ Microbiology Department, Shree M\& N Virani Science College, Rajkot, India \\ *corresponding author's email: shivangibhatt23@gmail.com
}

\begin{abstract}
The potential threats of bacterial resistance, especially widespread multi-drug resistance, are of major concern. The scenario of a patient dying from an infection caused by a multi-drug resistant organism is now a reality. Infections caused by resistant strains of bacteria are related to higher morbidity and mortality. $\beta$-Lactam antibiotics are a broad class of antibiotics, consisting of penicillin, cephalosporin, Monobactam, and carbapenems. $\beta$-lactam antibiotics are used to treat various infections. $\beta$-lactamases are enzymes responsible to provide resistance to $\beta$-lactam antibiotics. Among Gram-negative bacteria, the emergence of resistance to expanded-spectrum cephalosporin has been a major concern. Initially a limited number of bacterial species that could mutate to hyper produce their chromosomal class $\mathrm{C} \beta$-lactamase were found but now, resistance appeared in large number of bacterial species due to the production of TEM- or SHV-type ESBLs. In our study non clinical samples were selected to study the prevalence of drug resistance. After placing the various generations of $\beta$-lactam drugs we found that they were also resistant up to $4^{\text {th }}$ generation of $\beta$-lactam drugs. This study confirms the growing incidences of $\beta$-lactam resistant strains in bacterial species of community origin.
\end{abstract}

Key words: $\beta$-lactamase; Non clinical samples; Drug resistance

\section{Introduction}

The emergence of resistant strain is a recurrent problem that occurs from the introduction of antibiotics in clinical practice. Naturally occurring antibiotic resistance is very common. The genes that confer this resistance are known as the environmental resistome. These genes may be transferred from non-disease-causing bacteria to those that do cause disease, leading to clinically significant antibiotic resistance. (Wright GD, 2010)

$\beta$ lactam group of antibiotics such as penicillin, cephalosporin, Monobactam, and carbapenems represents more than $60 \%$ of all antimicrobials used worldwide. (Perilli et al. 2012) These molecules are preferred for their versatility, efficacy and safety. These drugs are effective against many gram-positive, gram-negative and anaerobic organisms.

Hospital-acquired bacterial infections may dominate the headlines, but most infections occur in the community. Indeed, $80 \%$ of the antibiotic prescribing takes place in the community - in local practices. (Varsha K Vaidya, 2011)

Bacterial resistance against beta-lactam antibiotics is increasing at a significant rate and has become a common problem in primary care medicine. There are several ways of antimicrobial resistance to beta-lactam antibiotics. (Holten K B., and Onusko E M., 2000.; McManus 1997; Tenover et al. 1998) One important mechanism is the production of beta-lactamases, which are enzymes, that breakdown the beta-lactam ring and inactivate the antibiotic. (Bush et al. 1995). The presence of these enzymes plays a crucial role in the selection of appropriate therapy.

The newer $\beta$-lactam antibiotics are found to be more effective in treating such type of resistant infections. The effectiveness of $\beta$-lactam drug is increased when they combine with $\beta$-lactamase inhibitor drugs such as clavulanic acid, tazobactam and salbactam.

Extended-spectrum b-lactamases (ESBLs) are plasmidmediated bacterial enzymes that confer resistance to a broad range of $\beta$-lactam. ESBLs are beta-lactamases that hydrolyze extended-spectrum cephalosporins with an oxyimino side chain. ESBL are dangerous because they are plasmid associated and the plasmid can exchange with variety of bacterial species. Conjugational transmission of antibiotic resistance genes across bacterial species and genera has amplified the problem of antibiotic resistance in pathogens. (Bhattacharjee et al. 2010). ESBL demonstrate resistance to $3^{\text {rd }}$ generation cephalosporin and so it is now a 
global threat and a matter of serious concern. (shakil et al., 2012)

ESBL's occur predominantly in Escherichia coli and Klebsiella species, they have also been described in other genera of the enterobacteriaceae (Bradford PA 2001, Paterson DL et al.. 2005), including Salmonella enterica, Pseudomonas aeruginosa, and Serratia marcescens (Morosini MI et al.., 1995, Naas T et al., 1999, Palucha A et al.., 1999)

Escherichia coli are important inhabitants of the human intestine. They are found in the faecal flora of almost all healthy adults. (Wilson, 2008). There are several toxin producing strains of $E$. coli causing diarrhoea, but most commonly these bacteria cause community onset UTIs. (Nataro et al. 2007)

ESBL producing enterobacteriaceae are an emerging public health concern (Pitout and Laupland, 2008), because the ESBL-encoding isolates often co express resistance to fluroquinolones, tetracycline and aminoglycosides, thus are often classified as multidrug-resistant pathogens (Paterson and Bonomo, 2005; Morosini et al., 2006).

This study was carried out to evaluate the $\beta$ - lactam drug resistance in non-clinical bacterial isolates. Different enterobacteriaceae were selected to check the prevalence of resistance towards various generations of $\beta$ - lactam drugs.

\section{Materials and methods}

The organisms were collected from soil, water and microbiology lab of educational institute (which were non clinical strain) for analysis. Ten different organisms were selected. These include Staphylococcus aureus, Salmonella typhi, Escherichia coli, Proteus vulgaris, Pseudomonas aeruginosa, Enterobacter aerogenes, Proteus Mirabilis, Klebsiella spp, Serretia marcescens and Shigella.

Antibiotic susceptibility was determined by Kirby Bauer disc diffusion method according to Clinical Laboratory Standard Institute (CLSI) guidelines. Antibiotics were chosen depending on the organism and the sample and results were interpreted as sensitive or resistant as per CLSI recommendations.

Various generation of $\beta$-lactam group of drugs were chosen for screening the $\beta$-lactam drug resistance. These includes

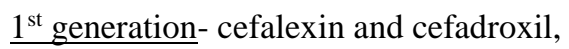

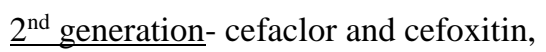

$\underline{3^{\text {rd }} \text { generation- }}$ ceftriaxone, cefotaxime, ceftazidime and cefoperazone,

$4^{\text {th }}$ generation- cefepime, carbapenems- ertapenem and imipenem, Monobactam- aztreonam

$\beta$-lactam/ $\beta$-lactamase inhibitor- cefotaxime and clavulanic acid, ceftazidime and clavulanic acid, ticarcillin and clavulanic acid, pipracillin and tazobactam, cefoperazone and salbactam, ampicillin and salbactam.

ESBLs were detected by the confirmatory method of National Committee for Clinical Laboratory Standards (NCCLS) now known as Clinical and Laboratory Standards Institute (CLSI) using cefotaxime $(30 \mathrm{mcg})$ and ceftazidime $(30 \mathrm{mcg})$ and a disc of cefotaxime plus clavulanic acid (30 $/ 10 \mathrm{mcg})$ and ceftazidime and clavulanic acid (30/10 mcg ) placed at a distance of $20 \mathrm{~mm}$ on a lawn culture $(0.5$ McFarland inoculum size) of suspected ESBL producing clinical isolate on Mueller-Hinton Agar (MHA, Hi-Media,) ESBL production was inferred if the inhibition zone increased by $5 \mathrm{~mm}$ towards the cefotaxime plus clavulanic acid disc or ceftazidime plus clavulanic acid disc in comparison to the third generation cephalosporin disc alone.

Various concentration of $3^{\text {rd }}$ and $4^{\text {th }}$ generation of antibiotics as well as $\beta$-lactam/ $\beta$-lactamase inhibitor drugs was used to check the spectrum of inhibition by cup plate method. The antibiotics selected were Cefotaxime, Aztreonam Cefetazidime+ Tazobactam and Cefepime+tazobactam. The concentration of antibiotics was $40 \mu \mathrm{g}, 50 \mu \mathrm{g}, 60 \mu \mathrm{g}$, $70 \mu \mathrm{g}, 80 \mu \mathrm{g}$ and $100 \mu \mathrm{g}$. After incubation the zone of inhibition of bacterial growth is measured with a millimetre scale.

\section{Result and Discussion}

The resistant organisms are now a worldwide problem in hospitals as well as in community samples. They can be found in a variety of Enterobacteriaceae species.

Knowledge of resistance pattern of bacterial strains in a geographical area will help to guide the health department for appropriate and judicious antibiotic use and to epidemiologist in the forecasting of the probable outbreak. There is possibility that the restricted use can lead to the withdrawal of selective pressure and resistant bacteria will no longer have survival advantage in such settings. (Mathur et al.. 2002)

The main purpose of the study was to evaluate the resistance towards $\beta$ - lactam group of drugs in non clinical strains which were generally found to be sensitive to most of the antibiotics. This can also throw light on the endemic diseases among the community members, laboratory technicians, and sudden outbreak of an infection on large scale in a community and the recurrence of a particular disease in a population.

Initial screening of organisms was done using various generations of $\beta$ - lactam group of drugs as shown in Fig. 1 . Total ten different $\beta$ - lactam drugs of various generations were selected. In this, we found majority of the strains showing resistance towards $2^{\text {nd }}$ generation and onwards $\beta$ lactam group of drugs. 

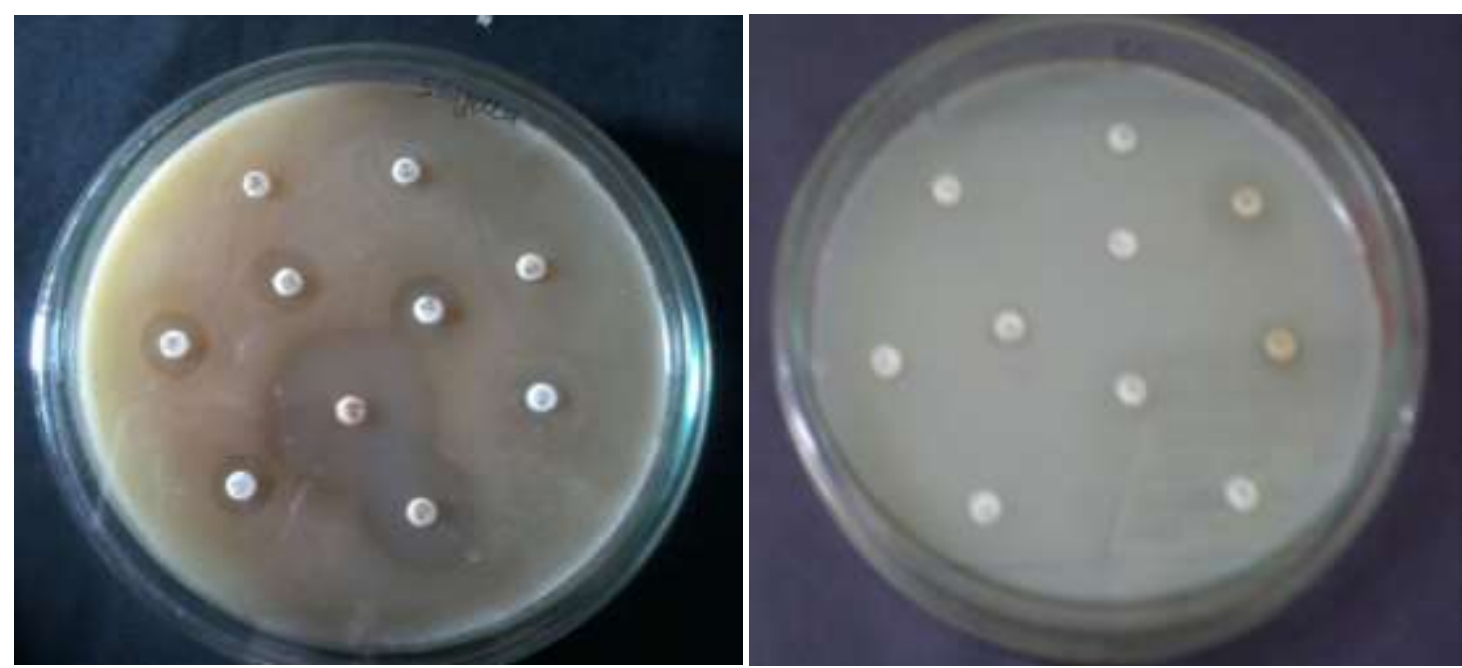

Fig. 1: The figure shows effect of various generations of $\beta$-lactam drugs on the growth of microbes.
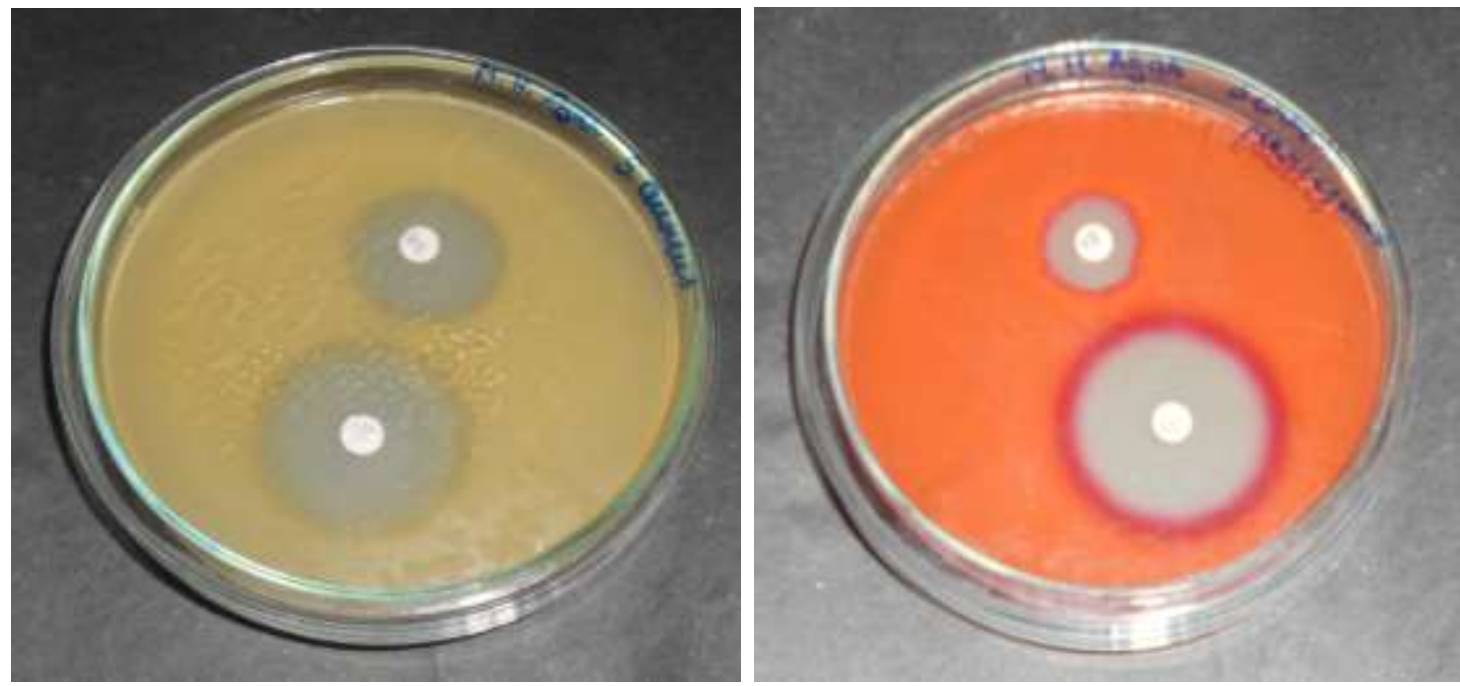

Fig. 2: The figure shows phenotypic detection of ESBL by placing 3rd generation $\beta$-lactam drug along with 3 rd generation $\beta$ lactam drug and combination of clavulanic acid.
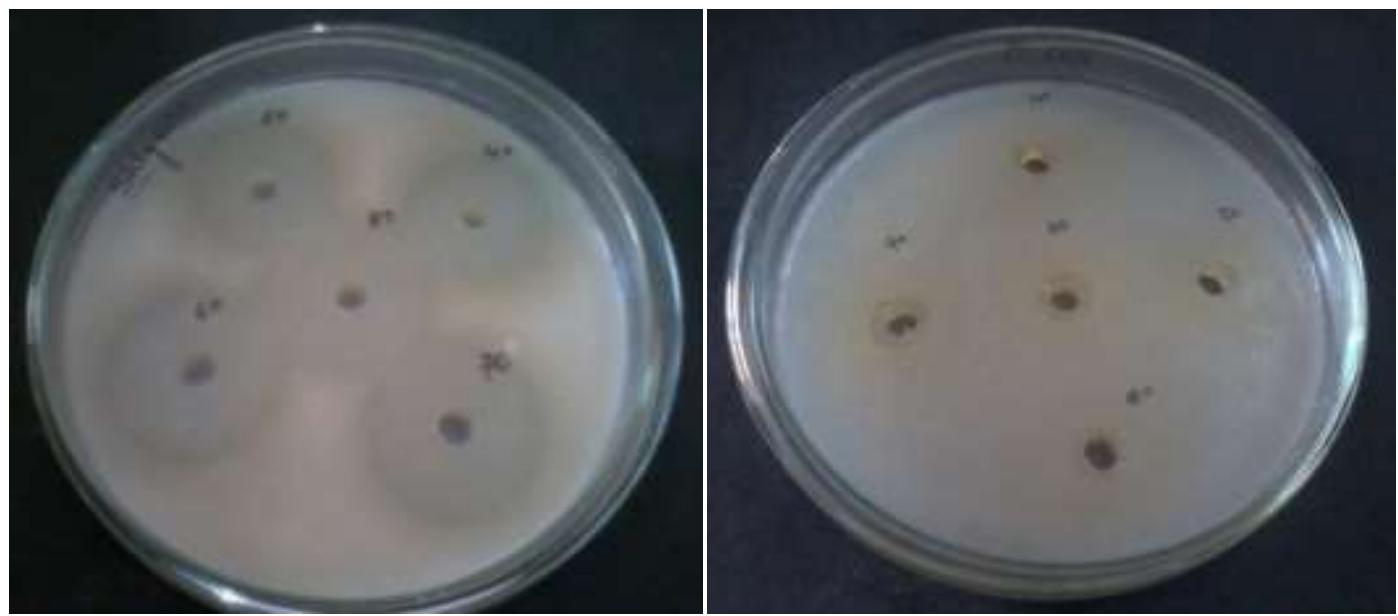

Fig. 3: Figure shows effect of different concentration of antibiotics by cup-plate method.

According to Fig. 4 the most common microbes which were resistant to many of these drugs were Escherichia coli, Proteus vulgaris, Pseudomonas aeruginosa and Shigella spp. Escherichia coli was found to be moderately sensitive towards ceftazidime and clavulanic acid combination drug only out of the ten drugs tested. Proteus vulgaris was sensitive towards cefotaxime, cefotaxime and clavulanic acid combination, imipenam and ceftriaxone only while Pseudomonas aeruginosa was resistant to all except carbapenems i.e. ertapenem and imipenam. 


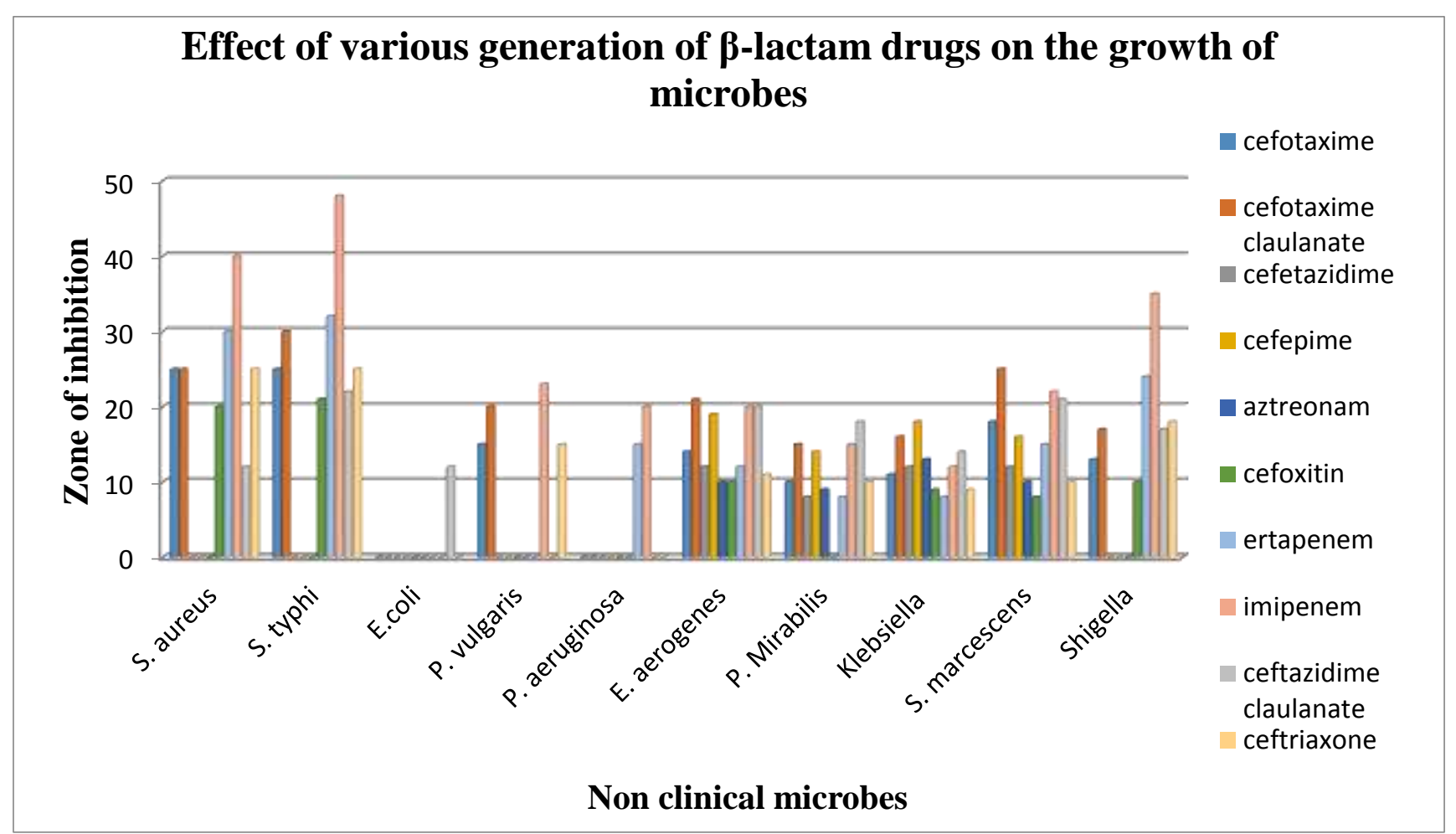

Fig. 4: The graph shows various generations of $\beta$-lactam drug and its effect on the growth of different non clinical microbes.

Major risk factors for acquisition of or infection with ESBL producing organisms are long term antibiotic exposure, prolonged ICU stay, nursing home residency, severe illness, residence in an institution with high rates of ceftazidime and other third generation cephalosporin use and instrumentation or catheterisation.(Nathisuwan et al., 2001)

The ESBL enzymes which are capable of hydrolyzing and inactivating a wide variety of $\beta$ lactams, including third generation cephalosporins, penicillins and aztreonam are plasmid coded enzymes. These are the result of mutations in TEM- 1 and TEM-2 and SHV-I. All of these $\beta$-lactamase enzymes are commonly found in the Enterobacteriaceae family. Generally, TEM-1, TEM-2 and SHV-1 enzymes confer high level resistance to early pencillins and low level resistance to first generation cephalosporins but widespread use of third generation cephalosporins and aztreonam is believed to be the major cause of the mutations in these enzymes that has led to the emergence of the ESBLs.

These enzymes mediate resistance to cefotaxime, ceftazidime and other broad spectrum cephalosporins and to monobactams such as aztreonam, but have no detectable activity against cephamycins and imipenem. Because, of their greatly extended substrate range these enzymes were called extended spectrum $\beta$-lactamases.(Sirot, 1995)

Majority of ESBL producing strains are $K$. pneumoniae, $K$. oxytoca and $E$. coli but other organisms reported to harbour ESBLs include Enterobacter spp., Salmonella, Morganella morganii, Proteus mirabilis, Serratia marcescens and Pseudomonas aeruginosa. However, the frequency of
ESBL production in these organisms is low. (Nathisuwan et al., 2001)

Although ESBL activity is inhibited by clavulanic acid the only infections that may be treated safely with $\beta$ lactam $\beta$ lactamase inhibitor combination are those involving the urinary tract. In this instance, b- lactamase inhibitor concentration is high enough to counteract the hydrolytic activity of ESBLs.(Nordmann, 1998)

Hence, we have performed the phenotypic detection of ESBL of the selected strains as shown in Fig. 2 and Fig. 5. According to Fig. 5 Pseudomonas aeruginosa was found to be highly resistant towards $3^{\text {rd }}$ generation cephalosporin as well as a combination of $\beta$-lactam/ $\beta$-lactamase inhibitor drugs. Other strains were found to be ESBL producing strains.

Fig. 3 shows the effect of different concentration $(40 \mu \mathrm{g}$, $50 \mu \mathrm{g}, 60 \mu \mathrm{g}, 70 \mu \mathrm{g}, 80 \mu \mathrm{g}$ and $100 \mu \mathrm{g}$ ) of antibiotics on selected microbes by cup-plate method.

Fig. 6 shows the graph of effect of various concentration of cefotaxime, a $3^{\text {rd }}$ generation $\beta$ lactam drug on the growth of selected microorganisms. According to this graph Escherichia coli and Pseudomonas aeruginosa were found to be highly resistant even up to $100 \mu \mathrm{g}$ concentration. Klebsiella was resistant up to $60 \mu \mathrm{g}$ concentration and then after was sensitive to higher concentrations. Proteus mirabilis shows sensitivity from $60 \mu \mathrm{g}$ concentration onwards. Rest of the organisms were found to be sensitive to all concentration of the drugs used. 


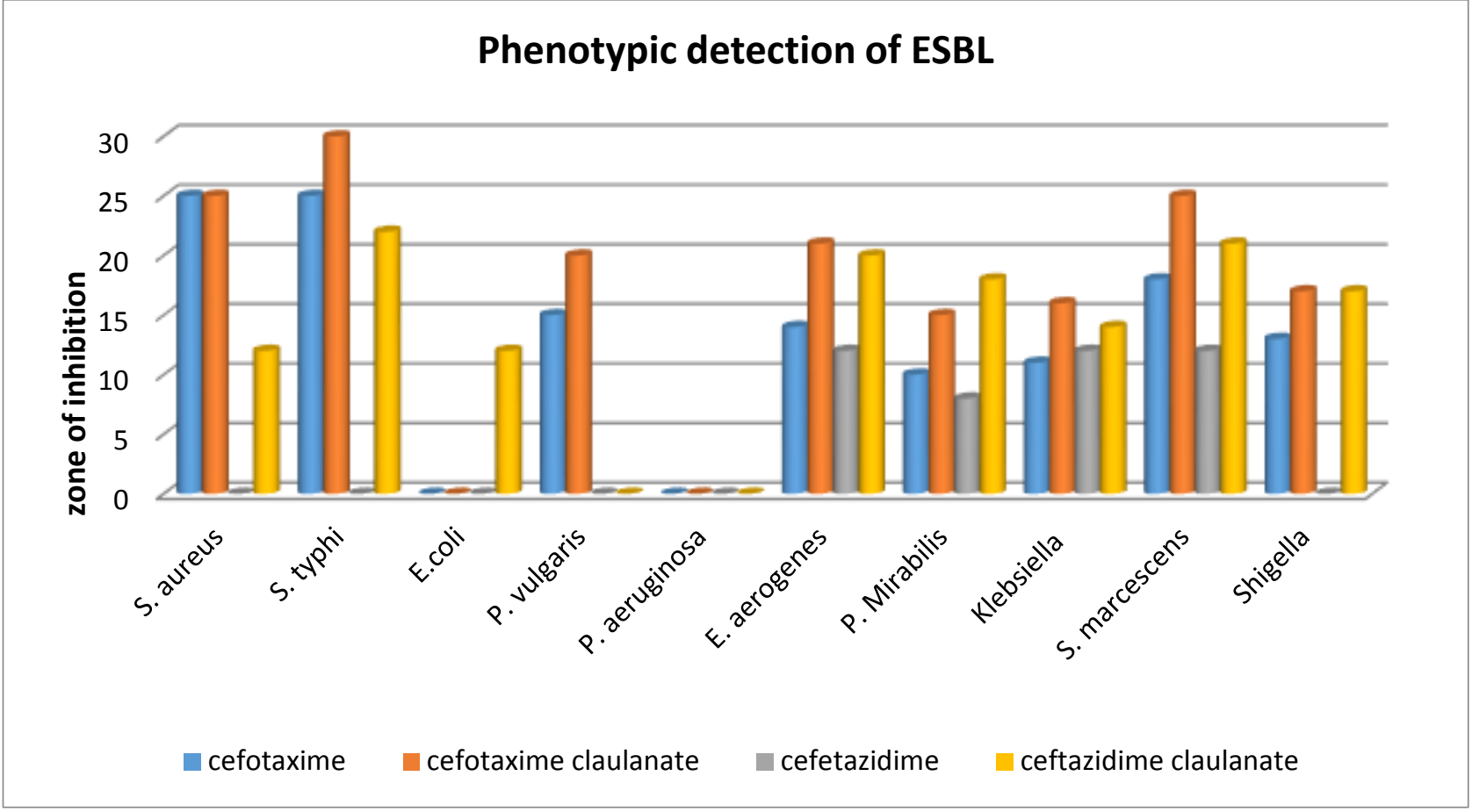

Fig. 5: The graph shows phenotypic detection of ESBL on different enterobacteriaceae.

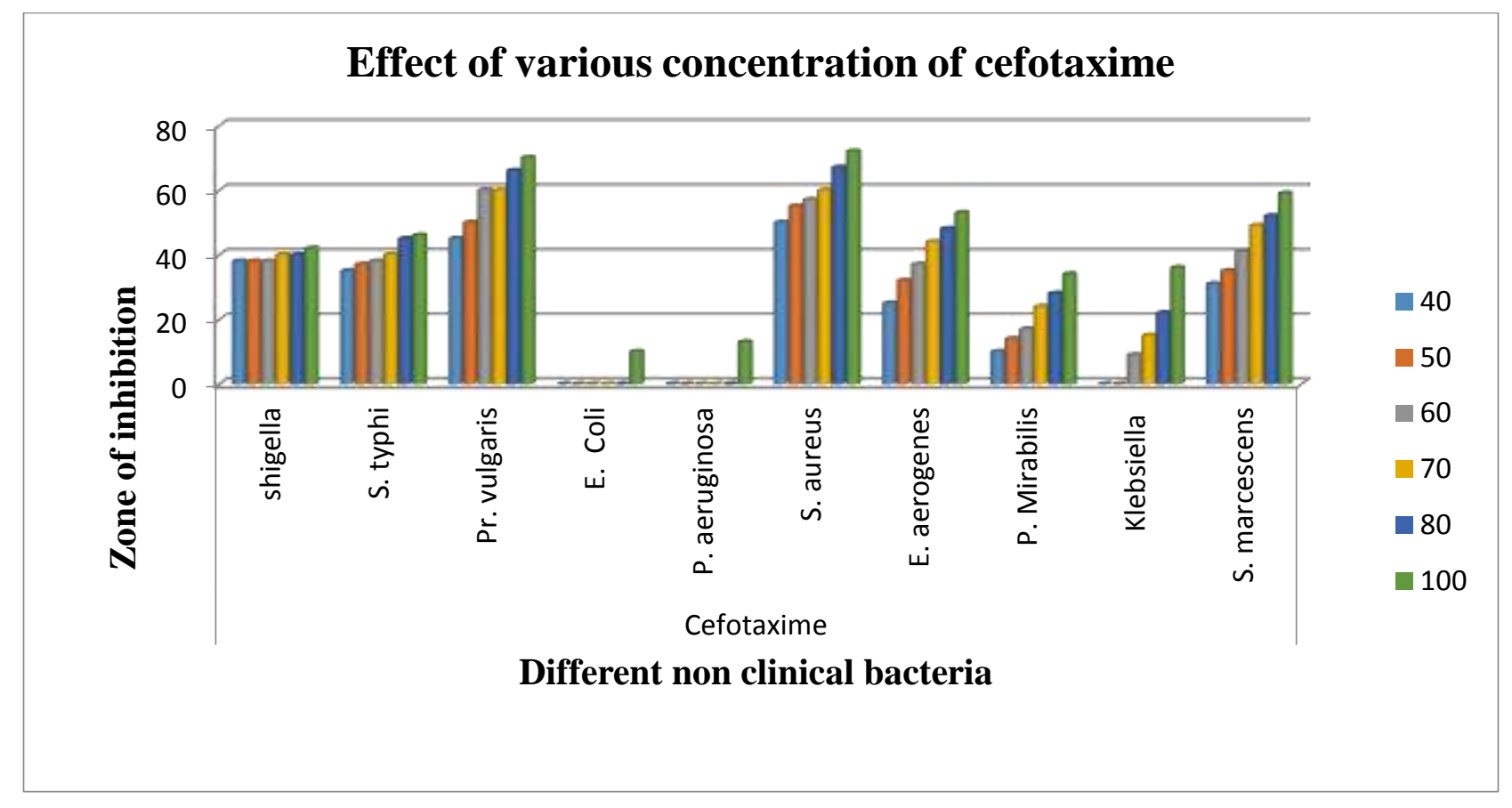

Fig. 6: The graph shows effect of various concentration of cefotaxime on the growth of microbes.

According to Fig. 7, which shows the effect of various concentration of a Monobactam drug i.e. aztreonam on the growth of microbes suggest that Escherichia coli, Salmonella, Proteus vulgaris and Staphylococcus aureus were found resistant at most of the concentrations and sensitive only at $100 \mu \mathrm{g}$ concentration. Enterobacter aerogenes, Proteus Mirabilis and Klebsiella spp, shows sensitivity from $60 \mu \mathrm{g}$ concentration and onwards. Pseudomonas aeruginosa, Serretia marcescens and Shigella were found to be sensitive.
Escherichia coli, Pseudomonas aeruginosa, and Proteus mirabilis are generally susceptible to aztreonam, while some staphylococci, Staphylococcus aureus, Staphylococcus hemolyticus and Xanthomonas maltophilia are resistant to it.( Mosby, 2006). But in our study Escherichia coli was found to be highly resistant.

In Fig. 8, the graph shows effect of various concentrations of ceftazidime + tazobactam $(\beta$-lactam/ $\beta$-lactamase inhibitor drug) on the growth of microbes. As this is a combination drug majority of the strains were found to be sensitive. 


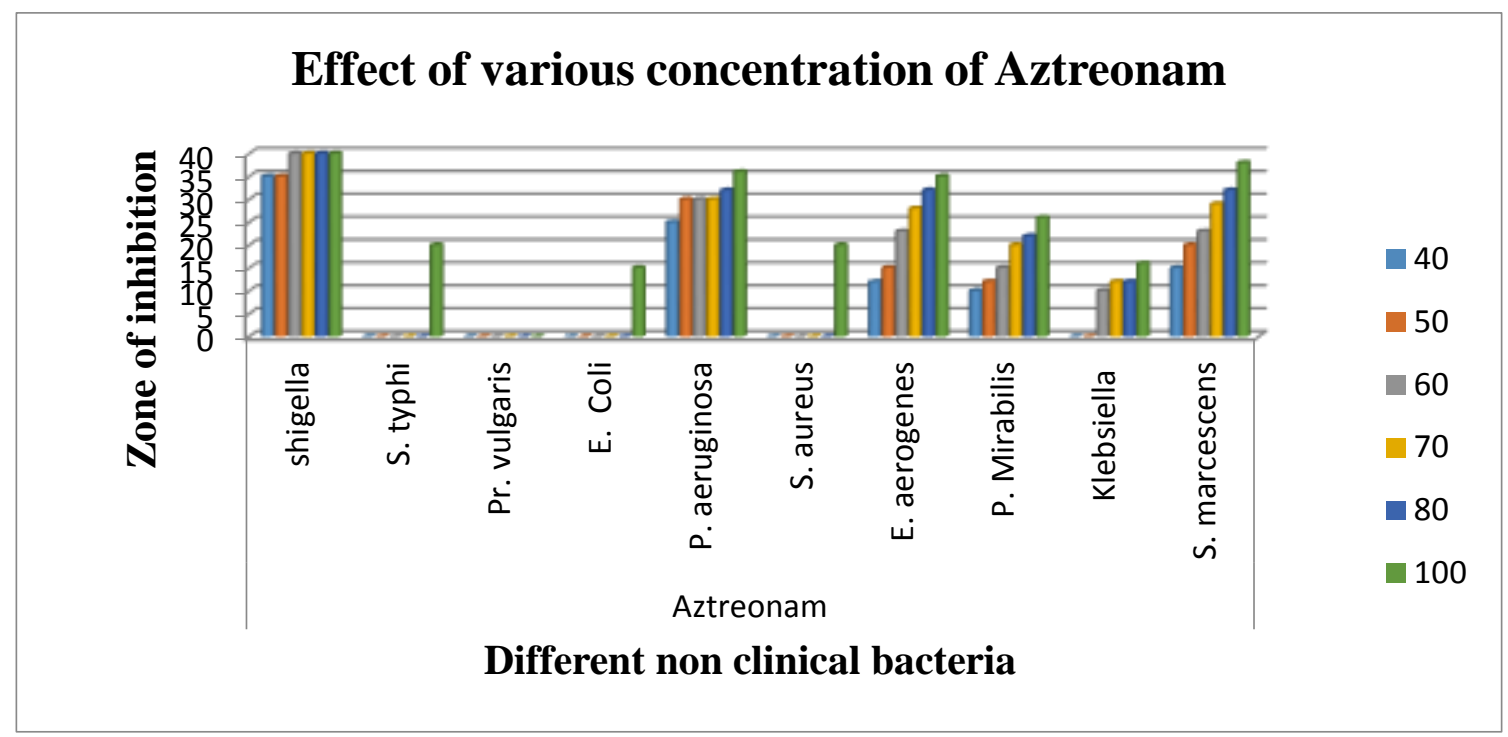

Fig 7: The graph shows effect of various concentration of aztreonam on the growth of microbes.

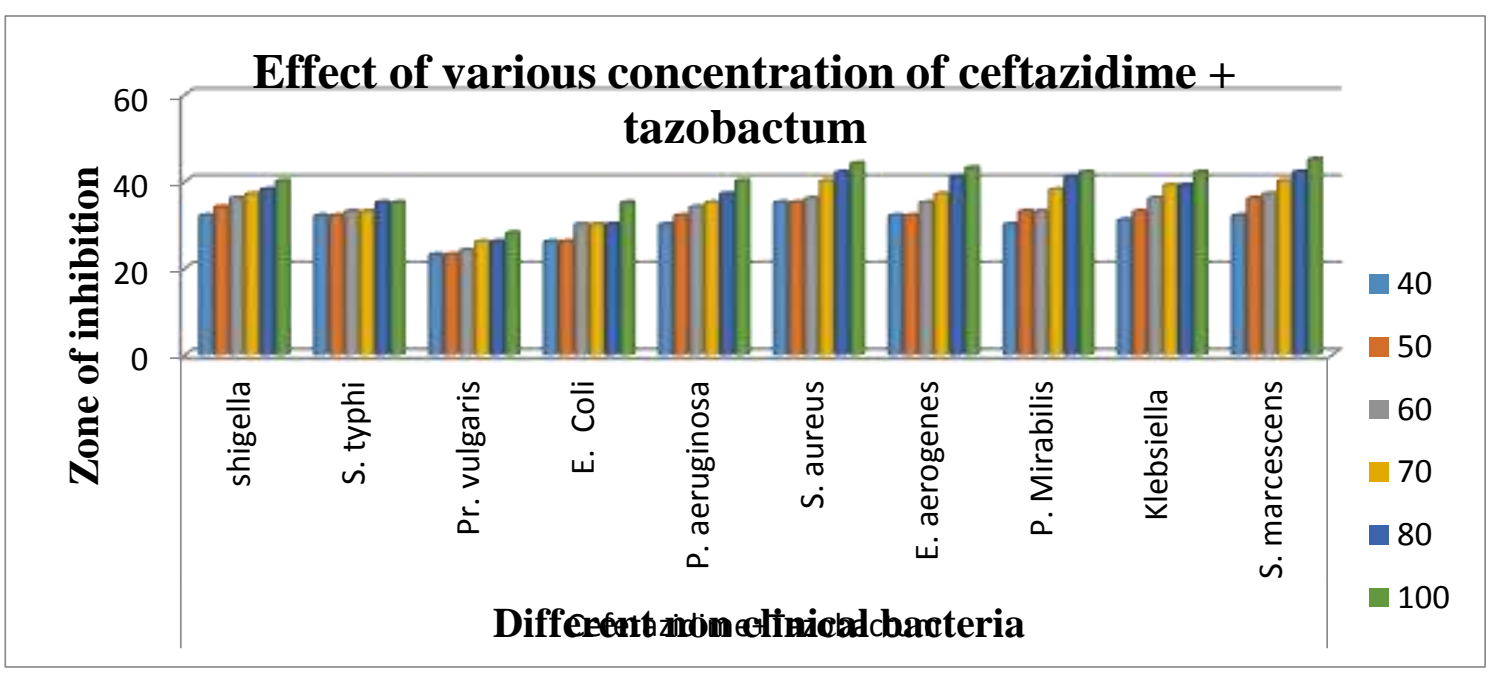

Fig 8: The graph shows effect of various concentrations of ceftazidime + tazobactam ( $\beta$-lactam/ $\beta$-lactamase inhibitor drug) on the growth of microbes.

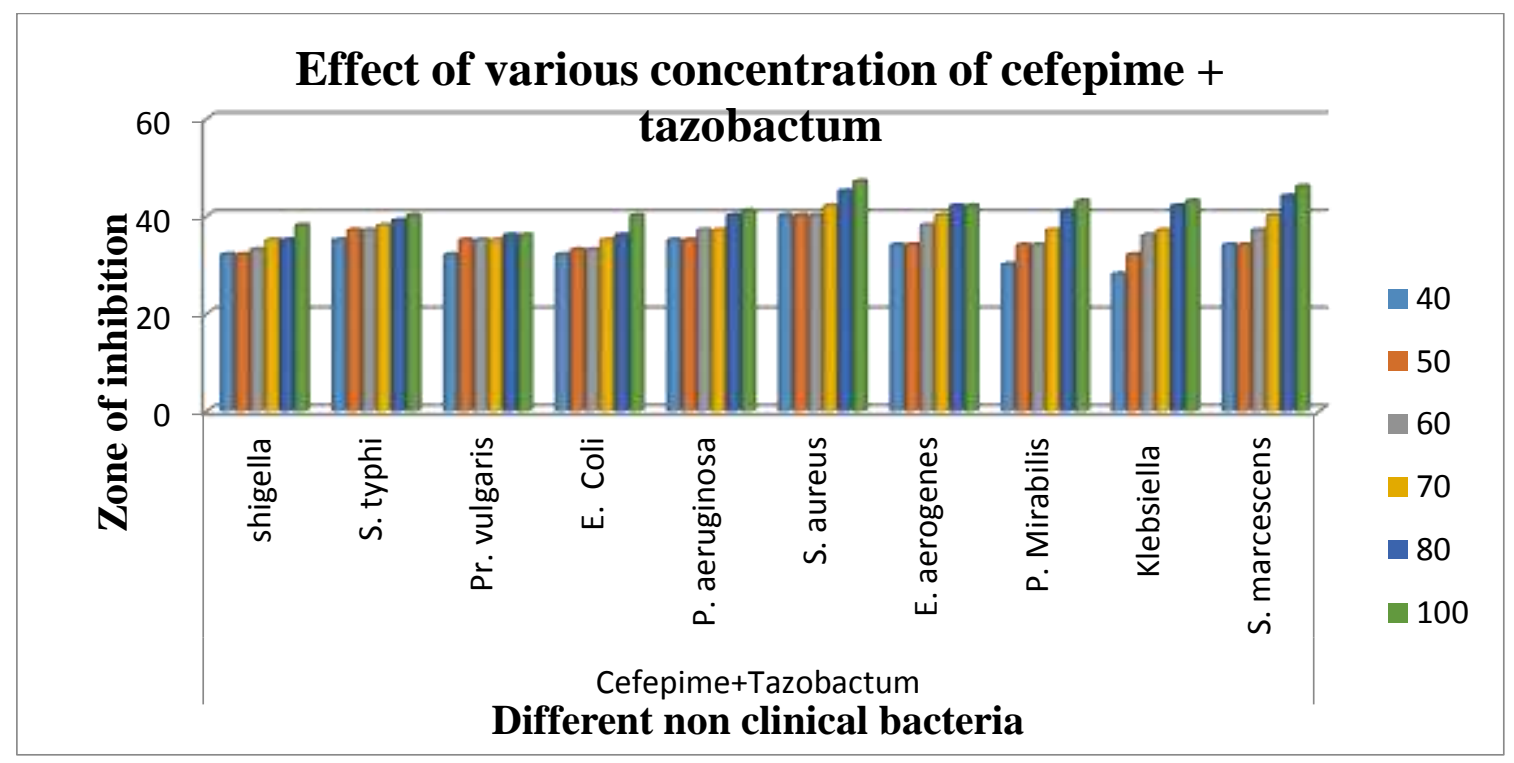

Fig 9: The graph shows effect of various concentrations of cefepime + tazobactam ( $\beta$-lactam/ $\beta$-lactamase inhibitor drug) on the growth of microbes.

Fig. 9 shows the $4^{\text {th }}$ generation $\beta$-lactam drug (cefepime) along with monobactam (tazobactam). This combination was also highly sensitive for the selected microorganism at all concentrations. 


\section{Conclusion}

$>$ Non clinical samples were selected to observe the prevalence of drug resistance. We found that the samples were resistant up to $4^{\text {th }}$ generation of $\beta$-lactam drugs. The drugs which were more effective are the carbapenams and combination drugs.

The incidence of infections due to organisms resistant to $\beta$-lactam agents due to production of various enzymes has increased in recent years.

$>$ Improper disposal of medical waste also contaminate the natural resources, which may be one of the reason for development of selective pressure on microbes.

$>$ Detection of ESBL production is of paramount importance both in hospital and community isolates. Firstly, ESBLs constitute a serious threat to currently available antibiotics. Secondly, institutional outbreaks are increasing because of selective pressure due to heavy use of expanded spectrum cephalosporin and lapses in effective control measures.

$>$ So we can conclude that antibiotic resistance is really a serious and big concern. If such emergence of superbugs will continue then antibiotics will be no more as 'magic bullets' to treat bacterial infections. Rather than giving single medication try to give combination therapy so chances or resistance can be reduced.

$>$ The extensive use of antibiotics in the community and hospitals has fuelled this crisis. Mechanisms such as antibiotic control programs, better hygiene, and synthesis of agents with improved antimicrobial activity need to be adopted in order to limit bacterial resistance.

\section{References}

Bhattacharjee, Sen MR, Prakash P, Gaur A, Anuprabha S, Nath G. (2010) Observation on integron carriage among clinical isolates of Klebsiella pneumonia producing extended spectrum $\beta$-lactamases. Indian journal of medical microbiology. 28(3): 207-210.

Bradford PA (2001) Extended-spectrum beta-lactamases in the 21st century: characterization, epidemiology, and detection of this important resistance threat. Clin. Microbiol. Rev. 14: 933-951.

Bush K, Jacoby GA, Medeiros AA (1995) A functional classification scheme for beta-lactamases and its correlation with molecular structure. Antimicrob Agents Chemother. 39:1211-1233.

Holten KB and Onusko EM (2000) Appropriate Prescribing of Oral Beta-Lactam Antibiotics. Am. Family Physician. 62(3): 611-620.

Mathur P, Kapil A, Das B and Dhawan B. (2002). Prevalence of ESBL producing gram negative bacteria in a tertiary care hospital. Indian J. Med. Res. 115:153-157.

McManus MC (1997) Mechanisms of bacterial resistance to antimicrobial agents. Am. J. Health Syst. Pharm. 54:1420-33.

Morosini MI, Canton R, Martinez-Beltran J, Negri MC, Perez-Diaz JC, Baquero F and Blazquez J (1995) New extended spectrum TEMtype $\beta$ - lactamase from Salmonella enterica subsp. enterica isolated in a nosocomial outbreak. Antimicrobial Agents Chemotherapy. 39: 458-461.

Morosini MI, Garcia- Castillo M, Coque TM, Valverde A, Novais A, Loza E, Baquero F and Canton R (2006) Antibiotic co resistance in extended-spectrum- $\beta$ lactamase producing Enterobacteriaceae and in vitro activity of tigecycline. Antimicrob. Agents Chemother. 50: 2695-2699.

Mosby (2006), Mosby's Drug Consult (Generic Prescription Physician's Reference Book Series.) Mosby; Inc. 16 edition

Naas T, Philippon L, Poirel L, Ronco E and Nordmann P (1999) An SHV-derived extended-spectrum blactamase in Pseudomonas aeruginosa. Antimicrob. Agents Chemother. 43:1281-4.

Nataro J, Bopp CA, Fields PI, Kaper JB and Stockbine NA (2007) Escherichia, Shigella, and Salmonella, In: Murray P, Baron EJ, Jorgensen JH, Landry ML and Pfaller MA (eds) Manual of clinical microbiology. Washington DC. ASM Press. p. 670-687.

Nathisuwan S, Burgess DS, Lewis II JS (2001) ESBLs: Epidemiology, Detection and Treatment. Pharmacotherapy 21(8): 920-928.

Nordmann P (1998) Trends in -lactam resistance among Enterobacteriaceae. Clin. Infect. Dis. 27(Suppl 1): S100-S1006.

Palucha A, Mikiewicz B, Hryniewicz W, Gniadkowski M. (1999) Concurrent outbreaks of extended-spectrum b-lactamase- producing organisms of the family Enterobacteriaceae in a Warsaw hospital. $J$. Antimicrob. Chemother. 44:489-99.

Paterson DL and Bonomo RA (2005) Extended-spectrum beta-lactamases: a clinical update. Clin. Microbiol. Rev. 18: 657-686. 
Perilli M, Celenza G, Pellegrini C and Amicosante G (2012) $\beta$ - lactamases a major mechanism of resistance in Gram negative bacteria. Multi drug resistance: A global concern, 210-226. Bentham science publishers.

Pitout JD, Laupland KB (2008) Extended-spectrum $\beta$ lactamase-producing Enterobacteriaceae: An emerging public-health concern. Lancet Infect Dis. 8: $159-166$.

Shakil S, Ali HM, Zarrilli R, Khan AU (2012) Extended spectrum beta lactamase: a critical update. Chapter 7. Multidrug resistance: a global concern. 115-129

Sirot D (1995) Extended - spectrum plasmid - mediated lactmases. J. Antimicrob. Chemother. 36(Suppl A): 19-34.
Tenover FC, McGowan JE (1998) The epidemiology of bacterial resistance to antimicrobial agents. In: Evans AS, Brachman PS, eds. Bacterial infections in humans: epidemiology and control. $3 \mathrm{~d}$ ed. New York: Plenum.

Varsha K Vaidya (2011) Horizontal Transfer of Antimicrobial Resistance by Extended-Spectrum $\beta$ Lactamase-Producing Enterobacteriaceae. J. Lab Physicians. 3(1): 37-42. DOI: 10.4103/09742727.78563

Wilson M (2008) Bacteriology of humans, an ecological perspective. Oxford. Blackwell Publishing.

Wright GD (2010) Antibiotic resistance in the environment: a link to the clinic? Current Opinion in Microbiology 13 (5): 589-594. 